\title{
Phosphorylation of PED/PEA-15 at Ser116 and phosphorylation of p27 at Thr187 indicates a poor prognosis in hepatocellular carcinoma
}

\author{
YIFENG WU ${ }^{1-3 *}$, XIANPENG LI $^{3 *}$, MINGLIANG $\mathrm{CHEN}^{3}, \mathrm{ZHIKUN} \mathrm{LIU}^{1,2}$, \\ XUANYU ZHANG ${ }^{1,2}$, SHUSEN ZHENG ${ }^{1,2}$ and XIAO XU ${ }^{1,2}$
}

\begin{abstract}
${ }^{1}$ Division of Hepatobiliary and Pancreatic Surgery, Department of Surgery, Collaborative Innovation Center for Diagnosis and Treatment of Infectious Diseases, The First Affiliated Hospital, Zhejiang University School of Medicine;

${ }^{2}$ Key Lab of Combined Multi-Organ Transplantation, Ministry of Public Health, Hangzhou, Zhejiang 310006;

${ }^{3}$ Division of Hepatobiliary and Pancreatic Surgery, Yinzhou Hospital Affiliated to Medical School of Ningbo University, Ningbo, Zhejiang 315040, P.R. China
\end{abstract}

Received December 24, 2019; Accepted November 24, 2020

DOI: $10.3892 / \mathrm{ol} .2021 .12438$

\begin{abstract}
Hepatocellular carcinoma (HCC) constitutes a deadly cancer with a high rate of recurrence and metastasis. Phosphoprotein enriched in diabetes/phosphoprotein enriched in astrocytes-15 (PED/PEA-15) is a protein involved in the metabolism of glucose that regulates numerous cellular processes, including cell division, apoptosis and migration in numerous types of cancer. However, PED/PEA-15 may act as a tumor-promotor or a tumor-suppressor depending on its phosphorylation status. In the present study, the association between the phosphorylation of PED/PEA-15 at Ser116 [PED/PEA-15(S116)], the phosphorylation of P27 at Thr187 [P-p27(T187)] and the clinicopathological features and prognosis of patients with HCC was assessed. The levels of PED/PEA-15(S116) and P-p27(T187) were determined using immunohistochemistry and western blotting analysis in resected liver tumor tissues and adjacent non-cancerous tissues obtained from 60 patients with HCC as well as normal liver tissues from 12 patients with benign lesions. The association between the expression levels of these two markers and the clinicopathological features of patients with HCC was
\end{abstract}

Correspondence to: Professor Xiao Xu, Division of Hepatobiliary and Pancreatic Surgery, Department of Surgery, Collaborative Innovation Center for Diagnosis and Treatment of Infectious Diseases, The First Affiliated Hospital, Zhejiang University School of Medicine, 79 Qingchun Road, Hangzhou, Zhejiang 310006, P.R. China

E-mail: zjxu@zju.edu.cn

*Contributed equally

Key words: hepatocellular carcinoma, phosphoprotein enriched in diabetes/phosphoprotein enriched in astrocytes-15 Ser116, phosphorylated p27 Thr187, prognostic biomarker explored. Using the Kaplan-Meier method, the prognostic value of PED/PEA-15(S116) and P-p27(T187) expression levels was determined. The results demonstrated that the levels of PED/PEA-15(S116) and P-p27(T187) proteins were remarkably higher in the HCC group compared with those in the adjacent and normal tissue groups (both $\mathrm{P}<0.05$ ). In addition, a moderate positive correlation was observed between the levels of PED/PEA-15(S116) and P-p27(T187) ( $r=0.434$; $\mathrm{P}<0.05)$. The levels of these two proteins were associated with the Edmondson grade, Tumor-Node-Metastasis (TNM) stage, vascular invasion and tumor multiplicity (all $\mathrm{P}<0.05$ ). Furthermore, the Kaplan-Meier analysis results demonstrated that patients with HCC that presented with positive expression of PED/PEA-15(S116) and P-p27(T187) exhibited a dismal prognosis compared with that in patients with negative expression regarding the overall survival (OS), as well as disease-free survival (both $\mathrm{P}<0.05)$. Multivariate Cox analysis revealed that the TNM stage $(\mathrm{P}<0.05)$, vascular invasion $(\mathrm{P}<0.05)$, PED/PEA-15(S116) levels $(\mathrm{P}<0.001)$ and $\mathrm{P}-\mathrm{p} 27$ (T187) levels $(\mathrm{P}<0.05)$ were independent prognostic factors for OS in patients with HCC. In conclusion the results of the present study demonstrated that PED/PEA-15(S116) and P-p27(T187) levels were upregulated in HCC tissues compared with those in the adjacent and normal tissues; PED/PEA-15(S116) and P-p27(T187) expression may serve as an indicator of a poor prognosis in patients with $\mathrm{HCC}$, suggesting that these proteins may be prospective therapeutic targets for HCC.

\section{Introduction}

Globally, hepatocellular carcinoma (HCC) constituted the third most common cause of cancer-related mortality in 2013 (1). Unlike the majority of other types of cancer, liver cancer mortality rates have increased by $34.8 \%$ in the USA over the past 20 years (2), suggesting that the liver cancer-associated health and economic burden will likely increase substantially worldwide in the coming decades (3). HCC is characterized by a poor prognosis, and the 5 -year survival rate is $\sim 15 \%$ (4). The 
main reasons contributing to the high mortality and poor prognosis include difficulties in early diagnosis and the complexity of tumor treatment in patients due to the vast heterogeneity of symptoms and tumor biology $(5,6)$. Therefore, identifying new prognostic markers that help to improve individual patient care is of great clinical significance.

Phosphoprotein enriched in diabetes/phosphoprotein enriched in astrocytes-15 (PED/PEA-15) is a ubiquitously expressed phosphoprotein in human glucose metabolism and was initially discovered in primary cultured astrocytes (7). PED/PEA-15 serves a key role in diabetes and glucose metabolism (8). In addition, PED/PEA-15 regulates a range of cellular processes, including cell proliferation, apoptosis and migration in numerous types of cancer, such as breast and lung cancer $(9,10)$. Notably, PED/PEA-15 acts as a tumor-promotor or a tumor-suppressor depending on its phosphorylation status (11). The unphosphorylated form binds extracellular signal receptor-activated kinase 1/2 (ERK1/2), suppressing its subsequent activation; by contrast, phosphorylation of PED/PEA-15 at Ser116 [PED/PEA-15(S116)] releases ERK1/2, resulting in its activation, which causes cell proliferation and migration, and thus, tumor promotion, including lung, breast and prostate cancer $(9,10,12)$. In addition, PED/PEA-15(S116) binds to the Fas-associated death domain protein (FADD), inhibiting FADD-mediated apoptosis (13).

The p27Kip1 (p27) protein regulates cellular functions such as differentiation, proliferation, apoptosis, translation and adhesion $(14,15)$. Previous evidence has demonstrated that the absence or low levels of p27 expression are indicators of a poor prognosis in several types of cancer, including colon, breast, bladder and lung cancer $(16,17)$. Notably, the major pathway of post-transcriptional nuclear degradation of p27 involves its phosphorylation at Thr187 [P-p27(T187)], which results in its suppression (18). During the $\mathrm{G}_{1} / \mathrm{S}$ phase transition of the cell cycle, P-p27(T187) allows the binding of S-phase kinase-associated protein 2 and the formation of cyclin E/A-Cdk2 complexes, leading to p27 ubiquitination and degradation $(19,20)$. Furthermore, ERK1/2 phosphorylation upregulates p27 phosphorylation and downregulates nuclear p27 levels (21). Despite the abundant information about PED/PEA-15(S116) and P-p27(T187), their relationship in HCC remains unclear. In addition, the association between PED/PEA-15(S116) and P-p27(T187) in a clinical setting has not been studied previously. Therefore, the present study aimed to explore the connection between PED/PEA-15(S116) and P-p27(T187) expression and the clinical features and prognosis in HCC.

\section{Materials and methods}

Patients and tissue specimens. A prospective single-center study was performed between January 2011 and January 2017. Samples for laboratory assays were obtained between January 2011 and December 2014. Tumor and adjacent non-tumorous hepatic $(2 \mathrm{~cm}$ from the edge of the tumor) tissues were harvested from 60 patients with HCC who had undergone hepatectomy by the same surgical team at Yinzhou Hospital Affiliated to Medical School of Ningbo University (Ningbo, China). The tissues were divided into two parts, one of which was immediately fixed with formalin and embedded in paraffin, whereas the other was stored in liquid nitrogen. Two independent hepatologists confirmed the diagnosis of HCC based on postoperative pathology. In addition, normal hepatic tissues were collected from 12 patients with benign lesions. No patient had received preoperative administration of neoadjuvant radiotherapy, chemotherapy or any invasive intervention, including percutaneous ablation or chemoembolization.

In the present study, the follow-up period was up to January 2017 or until the date of the patient's death. The data were collected by a telephone interview or outpatient service. Tumor-Node-Metastasis (TNM) stage and Edmondson grade were used to analyze the clinicopathological features in patients with HCC (22). The overall survival (OS) was determined as the period between the surgery date and death from any cause or the last follow-up date. The study received ethical approval from the Research Ethics Committee of Yinzhou Hospital (approval no. 2017006). Written informed consent was obtained from all participants of the present study. The protocols of the present study regarding human subjects followed the ethical standards of the National Research Committee and the Declaration of Helsinki.

Immunohistochemistry and evaluation of immunostaining. HCC, adjacent non-tumorous and normal tissue samples were fixed in a $10 \%$ neutral formalin solution immediately following resection at room temperature for $>24 \mathrm{~h}$. Dehydration was performed in an ethanol gradient series at room temperature as follows: $70 \%$ for $1 \mathrm{~h}, 80 \%$ for $2-4 \mathrm{~h}, 95 \%$ for $2-4 \mathrm{~h}, 95 \%$ for $2-4 \mathrm{~h}, 100 \%$ for $2-4 \mathrm{~h}$, and $100 \%$ for $2-4 \mathrm{~h}$. Subsequently the samples were embedded in paraffin, sectioned into 3-5- $\mu \mathrm{m}$ slices, mounted on glass slides and dewaxed. The slides were treated for $30 \mathrm{~min}$ with $1 \% \mathrm{H}_{2} \mathrm{O}_{2}$-methanol solution at room temperature and placed in citric acid/sodium citrate buffer $(0.01 \mathrm{M} ; \mathrm{pH} 6.0)$ at $92-98^{\circ} \mathrm{C}$, followed by microwaving for $10 \mathrm{~min}$ and washing three times for $5 \mathrm{~min}$ with PBS. Subsequently, the samples were incubated with $1 \%$ sheep serum (cat. no. ZLI-9021; Beijing Zhongshan Golden Bridge Biotechnology Co., Ltd.) in PBS to block non-specific antigens for $20 \mathrm{~min}$ at room temperature, and PED/PEA-15(S116) (1:50; cat. no. PA5-38314; Thermo Fisher Scientific, Inc.) and P-p27(T187) (1:50; cat. no. PA5-104911; Thermo Fisher Scientific, Inc.) primary antibodies were added in a dropwise manner and incubated at $4^{\circ} \mathrm{C}$ overnight. The following day, sheep anti-rabbit IgG-HRP horseradish peroxidase-conjugated secondary antibodies (1:1,000; cat. no. BHR101; Beijing Bersee Science and Technology Co., Ltd.) were added, followed by incubation at $37^{\circ} \mathrm{C}$ for $40 \mathrm{~min}$ in a humidified chamber. Subsequently, the avidin-biotin-peroxidase complex $(1: 100)$ (Beijing Bersee Science and Technology Co., Ltd.)) was added, and the slides were incubated at $37^{\circ} \mathrm{C}$ for $40 \mathrm{~min}$ in a humidified chamber. The samples were counterstained with 3,3'-diaminobenzidine at room temperature for $60-90 \mathrm{sec}$, dehydrated, cleared and sealed. PBS and normal sheep serum were used in place of the primary antibodies as the blank and negative controls, respectively. The staining results were observed under a light microscope with x10, x20 and x40 magnification (23). Similarly, the protein expression results were evaluated according to the frequency of positive staining in the cytoplasm or the nucleus of the cells (23). A 
Table I. Expression of PED/PEA-15(S116) and P-p27(T187) by immunohistochemistry in HCC, adjacent non-cancerous and normal tissue samples.

\begin{tabular}{|c|c|c|c|c|c|c|c|}
\hline \multirow[b]{2}{*}{ Variable } & \multirow[b]{2}{*}{$\mathrm{N}$} & \multicolumn{2}{|c|}{ Positive expression rate $(\%)$} & \multicolumn{2}{|c|}{$\chi^{2}$-value } & \multicolumn{2}{|c|}{$\mathrm{p}$-value } \\
\hline & & PED/PEA-15(S116) & P-p27(T187) & HCC vs. A & HCC vs. N & HCC vs. A & HCC vs. N \\
\hline $\mathrm{HCC}$ & 60 & $41(68.3)$ & $45(75.0)$ & & & & \\
\hline A & 60 & $20(33.3)$ & $21(35.0)$ & & & & \\
\hline $\mathrm{N}$ & 12 & $2(16.7)$ & $2(16.7)$ & & & & \\
\hline PED/PEA-15(S116) & & & & 14.70 & 11.09 & $<0.01$ & $<0.01$ \\
\hline P-p27(T187) & & & & 19.39 & 15.01 & $<0.01$ & $<0.01$ \\
\hline
\end{tabular}

PED/PEA-15(S116), phosphorylation of phosphoprotein enriched in diabetes/phosphoprotein enriched in astrocytes-15 at Ser116; P-p27(T187), phosphorylation of p27 at Thr187; HCC, hepatocellular carcinoma; A, adjacent non-cancerous liver tissue; N, normal liver tissue.

total of 10 different high magnification fields were randomly selected for double-blind counting (100 cells/field). Protein expression was categorized as positive when $>50 \%$ of the cells were stained.

Western blotting. Protein extraction and western blotting were performed following a standard protocol (24). Human frozen tissues (tumor tissues, adjacent non-tumorous and normal liver tissue) were crushed into a fine powder in a metal mortar, cooled on dry ice and lysed in RIPA lysis buffer $(50 \mathrm{mM}$ Tris- $\mathrm{HCl}, 150 \mathrm{mM} \mathrm{NaCl}, 1 \%$ Triton X-100, $1 \%$ sodium deoxycholate, $0.1 \%$ SDS, 1 mM PMSF) with 10:1 protease inhibitor cocktail (Sigma-Aldrich; Merck KGaA). The protein concentration was determined by the BCA assay. The extracted proteins were separated by SDS-PAGE (40 $\mu \mathrm{g}$ protein per lane; $12 \%$ separation gel and 5\% spacer gel) and transferred to PVDF membranes. Following blocking with TBS-0.1\% Tween-20 (TBS-T) buffer containing $5 \%$ non-fat powder milk at room temperature for $2 \mathrm{~h}$, the membranes were immunoblotted using the following primary antibodies: Anti- $\beta$-actin (1:200; cat. no. sc-8432; Santa Cruz Biotechnology, Inc.), anti-PED/PEA-1 5 (1:800; cat. no. PA5-100749; Thermo Fisher Scientific, Inc.), anti-PED/PEA-15(S116) (1:800; cat. no. PA5-38314; Thermo Fisher Scientific, Inc.), anti-P27 (1:800; cat. no. PA5-27188; Thermo Fisher Scientific, Inc.) and anti-P-p27(T187) (1:800; cat. no. PA5-104911; Thermo Fisher Scientific, Inc.), at $4^{\circ} \mathrm{C}$ for $>12 \mathrm{~h}$, and the goat anti-mouse $\mathrm{IgG}$ peroxidase-conjugated secondary antibody (1:800; Pierce; Thermo Fisher Scientific, Inc.) was added and incubated in a $37^{\circ} \mathrm{C}$ water bath for $1 \mathrm{~h}$. The membranes washed three times with TBS-T. Immunoreactive bands on the blots were visualized with an ECL kit (Beit HaEmek).

Statistical analysis. The data were analyzed using SPSS 22.0 software (IBM Corporation). Continuous variable values were expressed as the mean \pm standard error of the mean and analyzed using Student's t-test. The analysis of multiple groups was performed by one-way ANOVA with Tukey's post hoc test. Categorical variables were compared using the $\chi^{2}$ or Fisher's exact test. In addition, Spearman's rank correlation analysis was used to analyze the associations between
PED/PEA-15(S116) and P-p27(T187). Survival curves were drawn using the Kaplan-Meier method and analyzed by the log-rank test. Multivariate analysis was performed on the prognostic variables using the Cox proportional hazard regression model with stepwise forward selection. $\mathrm{P}<0.05$ was considered to indicate a statistically significant difference.

\section{Results}

Detection of PED/PEA-15(S116) and P-p27(T187) protein expression. The immunohistochemical staining results revealed positive staining for both PED/PEA-15(S116)-and P-p27(T187) in the cytoplasm. (Fig. 1). The rates of positive expression for PED/PEA-15(S116) and P-p27(T187) in patients with HCC were $68.3 \%$ (41/60) and 75.0\% (45/60), respectively. These rates were higher compared with those in the adjacent [PED/PEA-15(S116), 33.3\% (20/60), P<0.05; P-p27(T187), $35 \%(21 / 60), \mathrm{P}<0.05]$ and normal tissues [PED/PEA-15(S116), $16.7 \%$ (2/12), $\mathrm{P}<0.05$; $\mathrm{P}-\mathrm{p} 27$ (T187), 16.7\% (2/12), $\mathrm{P}<0.05$; Table I]. Western blot analysis revealed higher levels of P-p27(T187) in the HCC group compared with those in the adjacent and normal liver tissue groups. In addition, the expression levels of PED/PEA-15 and PED/PEA-15(S116) in the HCC group were higher compared with those in the adjacent and normal liver tissue groups. (Fig. 2; Table II).

Associations between PED/PEA-15(S116) and P-p27(T187) expression and clinicopathological features in patients with HCC. As presented in Table III, the PED/PEA-15(S116) expression levels were associated with the Tumor-Node-Metastasis (TNM) stage $(\mathrm{P}<0.05)$, Edmondson grade $(\mathrm{P}<0.05)$, vascular invasion $(\mathrm{P}<0.05)$ and tumor multiplicity $(\mathrm{P}<0.05)$. Significant associations between P-p27(T187) expression levels and TNM stage $(\mathrm{P}<0.05)$, Edmondson grade $(\mathrm{P}<0.05)$, vascular invasion $(\mathrm{P}<0.05)$ and tumor multiplicity $(\mathrm{P}<0.05)$ were also identified. The expression levels of PED/PEA-15(S116) and P-p27(T187) were not associated with patient age, sex, tumor size, cirrhosis, $\alpha$-fetoprotein level or hepatitis B surface antigen positivity (all $\mathrm{P}>0.05$ ).

Correlation between the expression of PED/PEA-15(S116) and P-p27(T187) in HCC. The correlation between 


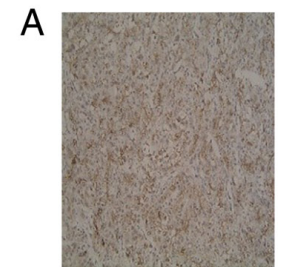

$\mathrm{T}$

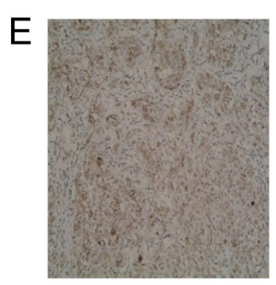

$\mathrm{T}$

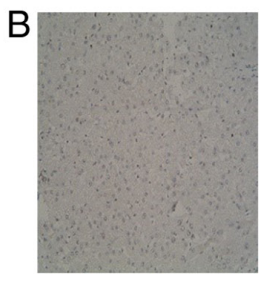

A

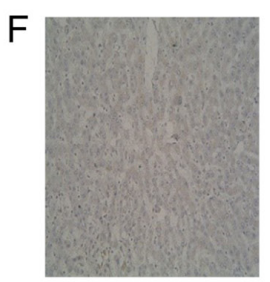

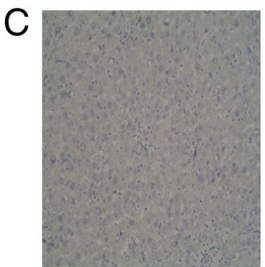

N

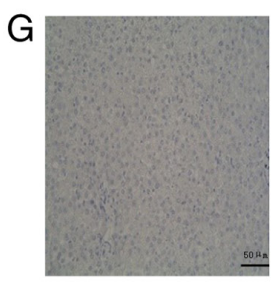

$\mathrm{N}$
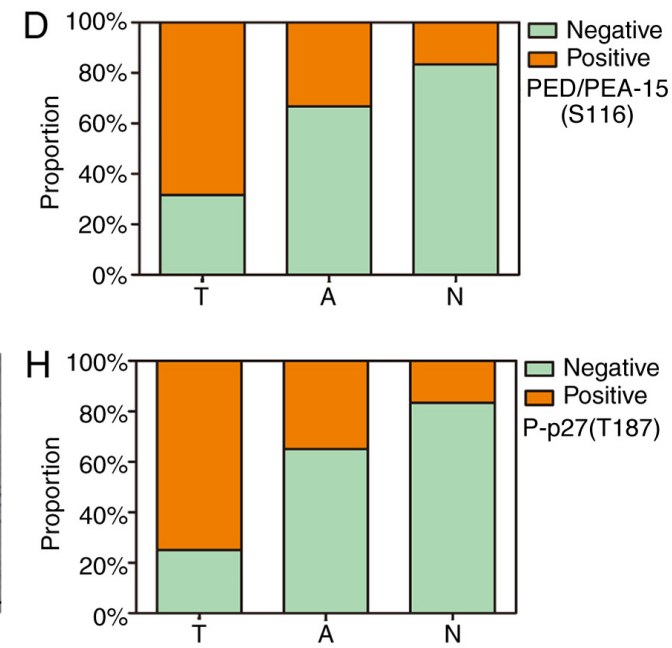

Figure 1. Representative images of PED/PEA-15(S116) and P-p27(T187) expression. (A-D) PED/PEA-15(S116) protein expression levels were higher in HCC tissues compared with those in the adjacent and normal tissue samples, as determined using immunohistochemistry. (A) High PED/PEA-15(S116) expression in HCC tissue. (B) Low PED/PEA-15(S116) expression in adjacent non-cancerous tissue. (C) No PED/PEA-15(S116) expression in normal liver tissue. (E-H) P-p27(T187) protein expression levels were higher in HCC tissues compared with those in the adjacent non-cancerous and normal tissue samples. (E) High P-p27(T187) expression in HCC tissue. (F) Low P-p27(T187) expression in adjacent non-cancerous tissue. (G) No P-p27(T187) expression in normal liver tissue. Scale bar, $50 \mu \mathrm{m}$. PED/PEA-15(S116), phosphorylation of phosphoprotein enriched in diabetes/phosphoprotein enriched in astrocytes-15 at Ser116; P-p27(T187), phosphorylation of p27 at Thr187; HCC, hepatocellular carcinoma; T, HCC group; A, adjacent non-cancerous group; N, normal group.

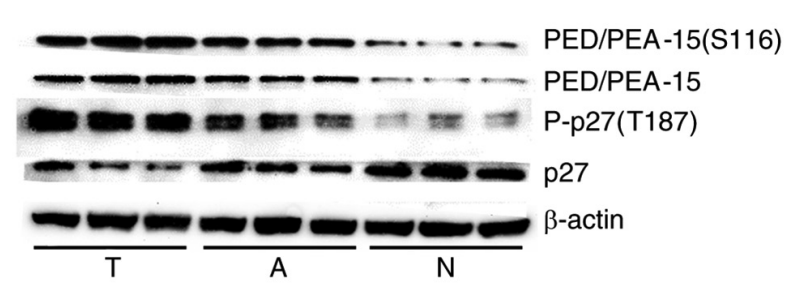

Figure 2. Representative images of total and phosphorylated PED/PEA-15 and P27 protein expression levels determined by western blotting. PED/PEA-15, phosphoprotein enriched in diabetes/phosphoprotein enriched in astrocytes-15; PED/PEA-15(S116), phosphorylation of PED/PEA-15 at Ser116; P-p27(T187), phosphorylation of p27 at Thr187; T, hepatocellular carcinoma group; A, adjacent non-cancerous group; N, normal group.

PED/PEA-15(S116) and P-p27(T187) expression was evaluated in HCC tissues. The Spearman rank correlation analysis results revealed that the expression of PED/PEA-15(S116) was positively associated with $\mathrm{P}-\mathrm{p} 27(\mathrm{~T} 187)$ expression $(\mathrm{r}=0.434$; $\mathrm{P}<0.05$ ) (Table IV).

Association of PED/PEA-15(S116) and P-p27(T187) expression with clinical prognosis. Kaplan-Meier survival analysis were performed using data from the 60 patients with HCC to determine the effects of PED/PEA-15(S116) and P-p27(T187) protein expression on patient prognosis. The results revealed that the patients with HCC with positive PED/PEA-15(S116) or P-p27(T187) expression exhibited shorter OS times (Fig. 3C, $\mathrm{P}<0.05)$. Similar trends were observed for disease-free survival, where patients with HCC with positive PED/PEA-15(S116) or P-p27(T187) expression exhibited shorter disease-free survival times compared with those with negative expression (Fig. 3D, P<0.05). In addition, the effects of concomitant positive expression of P-p27(T187) and PED/PEA-15 on OS and disease-free survival time were also evaluated. The results revealed that patients with $\mathrm{HCC}$ with positive expression of P-p27(T187) and PED/PEA-15(S116) exhibited a poorer prognosis compared with those with positive expression of P-p27(T187) or PED/PEA-15(S116) alone (P<0.05, Fig. 4).

Univariate and multivariate analyses of prognostic variables in HCC. Univariate and multivariate analyses were performed to assess whether PED/PEA-15(S116) and P-p27(T187) expression constituted an independent risk factor for HCC outcomes. The results demonstrated that age, sex, serum $\alpha$-fetoprotein level, hepatitis B surface antigen positivity, tumor size, multiplicity, Edmondson grade, TNM stage, vascular invasion and positive expression of PED/PEA-15(S116) and P-p27(T187) were identified as prognostic variables for the $\mathrm{OS}$ rate in patients with HCC. In the multivariate analysis, only TNM stage $(\mathrm{P}<0.05)$, vascular invasion $(\mathrm{P}<0.05), \mathrm{PED} / \mathrm{PEA}-15(\mathrm{~S} 116)$ expression $(\mathrm{P}<0.05)$ and $\mathrm{P}-\mathrm{p} 27(\mathrm{~T} 187)$ expression $(\mathrm{P}<0.05)$ were identified as independent prognostic variables for $\mathrm{HCC}$ OS (Table V).

\section{Discussion}

In the present study, the expression levels of PED/PEA-15(S116) and P-p27(T187) were assessed in HCC, adjacent non-tumor and normal liver tissues. In addition, the associations between PED/PEA-15(S116) and P-p27(T187) expression levels, clinicopathological features and clinical outcomes of patients with HCC were analyzed. The results demonstrated that PED/PEA-15(S116) and P-p27(T187) expression levels were higher in HCC tissues compared with those in adjacent and normal liver tissues. In addition, the expression of PED/PEA-15(S116) was associated with the expression levels of P-p27(T187) in HCC tissues, as determined by Spearman rank correlation analysis. High expression levels of these markers were associated with TNM stage, Edmondson grade, vascular invasion and tumor multiplicity. Kaplan-Meier survival analysis results demonstrated that patients with $\mathrm{HCC}$ with positive 
Table II. Expression of PED/PEA-15(S116), P-p27(T187), PED/PEA-15, and P27 by western blotting in HCC, adjacent non-cancerous and normal tissue samples.

t-value

P-value

Variable

$\mathrm{HCC}$

A

$\mathrm{N}$

HCC vs. A HCC vs. N

HCC vs. A

HCC vs. N

N

OD ratio, mean \pm SEM

PEA-15(S116)/actin

P-p27(T187)/actin

PEA-15/actin

P27/actin

PEA-15(S116)/PEA-15

P-p27(T187)/P27
60

$0.64 \pm 0.06$
$0.64 \pm 0.11$
$0.62 \pm 0.08$
$0.21 \pm 0.06$
$1.02 \pm 0.14$
$3.03 \pm 0.31$

60

$\begin{array}{llr}0.35 \pm 0.07 & 0.18 \pm 0.04 & 17.80 \\ 0.31 \pm 0.08 & 0.15 \pm 0.03 & 16.38 \\ 0.35 \pm 0.08 & 0.19 \pm 0.07 & 16.55 \\ 0.34 \pm 0.08 & 0.66 \pm 0.14 & -10.58 \\ 1.00 \pm 0.21 & 0.94 \pm 0.05 & 0.84 \\ 0.91 \pm 0.20 & 0.23 \pm 0.05 & 42.21\end{array}$

$0.23 \pm 0.05$
12

42.21

22.66
23.23
17.51
-12.54
0.52
28.48

22.66

0.01

$<0.01$

$<0.01$

$<0.01$

0.04

$<0.01$

$<0.01$
$<0.01$
0.01
$<0.01$
0.02
$<0.01$

PED/PEA-15, phosphoprotein enriched in diabetes/phosphoprotein enriched in astrocytes-15; PED/PEA-15(S116), phosphorylation of PED/PEA-15 at Ser116; P-p27(T187), phosphorylation of p27 at Thr187; HCC, hepatocellular carcinoma; A, adjacent non-cancerous liver tissue; N, normal liver tissue; OD, optical density.
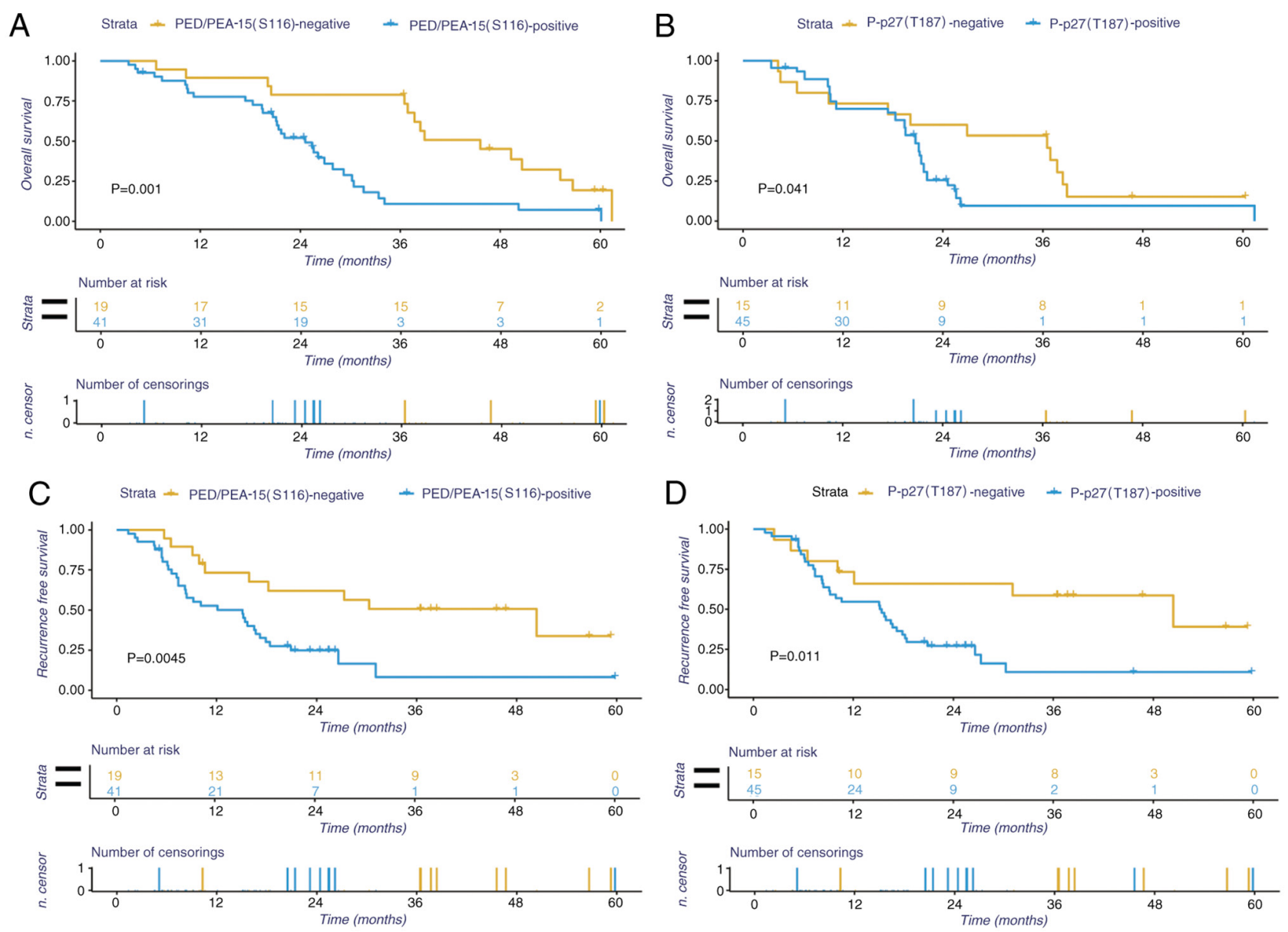

Figure 3. Prognostic value of PED/PEA-15(S116) and P-p27(T187) expression in patients with HCC. (A and B) Kaplan-Meier analysis demonstrated differences in overall survival between patients with HCC ( $n=60)$ with positive and negative (A) PED/PEA-15(S116) and (B) P-p27(T187) expression. (C and D) A similar trend was observed for disease-free survival in patients with HCC with positive and negative (C) PED/PEA-15(S116) and (D) P-p27(T187) expression. PED/PEA-15(S116), phosphorylation of phosphoprotein enriched in diabetes/phosphoprotein enriched in astrocytes-15 at Ser116; P-p27(T187), phosphorylation of p27 at Thr187; HCC, hepatocellular carcinoma.

expression of PED/PEA-15(S116) and P-p27(T187) exhibited poorer clinical outcomes compared with those in patients with negative expression. Multivariate survival analysis results demonstrated that the expression levels of PED/PEA-15(S116) 
Table III. Associations between PED/PEA-15(S116) and P-p27(T187) expression levels determined by western blotting and the clinicopathological characteristics of patients with hepatocellular carcinoma $(n=60)$.

OD value of protein expression (mean \pm SEM)

\begin{tabular}{|c|c|c|c|c|c|}
\hline & & & & & \\
\hline Variables & $\mathrm{N}$ & $\mathrm{PED} / \mathrm{P}$ & & $\mathrm{P}-\mathrm{p}$ & \\
\hline Age, years & & & $\mathrm{P}=0.775$ & & $\mathrm{P}=0.769$ \\
\hline$\leq 50$ & 27 & $0.598 \pm 0.017$ & & $0.625 \pm 0.022$ & \\
\hline$>50$ & 33 & $0.604 \pm 0.016$ & & $0.617 \pm 0.019$ & \\
\hline Sex & & & $\mathrm{P}=0.341$ & & $\mathrm{P}=0.795$ \\
\hline Male & 37 & $0.618 \pm 0.014$ & & $0.629 \pm 0.018$ & \\
\hline Female & 23 & $0.596 \pm 0.010$ & & $0.623 \pm 0.020$ & \\
\hline HBsAg & & & $\mathrm{P}=0.175$ & & $\mathrm{P}=0.149$ \\
\hline Positive & 45 & $0.602 \pm 0.018$ & & $0.624 \pm 0.011$ & \\
\hline Negative & 15 & $0.571 \pm 0.024$ & & $0.591 \pm 0.022$ & \\
\hline Tumor size, $\mathrm{cm}$ & & & $\mathrm{P}=0.099$ & & $\mathrm{P}=0.145$ \\
\hline$\leq 5$ & 38 & $0.569 \pm 0.018$ & & $0.612 \pm 0.019$ & \\
\hline$>5$ & 22 & $0.618 \pm 0.021$ & & $0.658 \pm 0.025$ & \\
\hline Cirrhosis & & & $\mathrm{P}=0.083$ & & $\mathrm{P}=0.142$ \\
\hline Yes & 42 & $0.615 \pm 0.014$ & & $0.645 \pm 0.015$ & \\
\hline No & 18 & $0.566 \pm 0.026$ & & $0.599 \pm 0.031$ & \\
\hline Tumor multiplicity & & & $\mathrm{P}=0.004$ & & $\mathrm{P}=0.024$ \\
\hline Single & 39 & $0.538 \pm 0.019$ & & $0.587 \pm 0.019$ & \\
\hline Multiple & 21 & $0.625 \pm 0.021$ & & $0.667 \pm 0.029$ & \\
\hline $\mathrm{AFP}, \mu \mathrm{g} / \mathrm{l}$ & & & $\mathrm{P}=0.075$ & & $\mathrm{P}=0.112$ \\
\hline$\leq 400$ & 23 & $0.594 \pm 0.019$ & & $0.626 \pm 0.028$ & \\
\hline$>400$ & 37 & $0.632 \pm 0.012$ & & $0.680 \pm 0.019$ & \\
\hline Edmondson grade & & & $\mathrm{P}=0.005$ & & $\mathrm{P}=0.005$ \\
\hline I-II & 32 & $0.577 \pm 0.019$ & & $0.595 \pm 0.020$ & \\
\hline III-IV & 28 & $0.644 \pm 0.013$ & & $0.676 \pm 0.019$ & \\
\hline TNM stage & & & $\mathrm{P}=0.013$ & & $\mathrm{P}=0.001$ \\
\hline I-II & 38 & $0.578 \pm 0.017$ & & $0.597 \pm 0.017$ & \\
\hline III & 22 & $0.648 \pm 0.020$ & & $0.695 \pm 0.024$ & \\
\hline Vascular invasion & & & $\mathrm{P}=0.005$ & & $\mathrm{P}=0.009$ \\
\hline Yes & 19 & $0.655 \pm 0.015$ & & $0.699 \pm 0.021$ & \\
\hline No & 41 & $0.585 \pm 0.013$ & & $0.616 \pm 0.019$ & \\
\hline
\end{tabular}

PED/PEA-15(S116), phosphorylation of phosphoprotein enriched in diabetes/phosphoprotein enriched in astrocytes-15 at Ser116; P-p27(T187), phosphorylation of p27 at Thr187; HBsAg, hepatitis B virus surface antigen; AFP, $\alpha$-fetoprotein; TNM, tumor-node-metastasis; OD, optical density.

and P-p27(T187) were independent prognostic indicators for patients with HCC.

HCC continues to be a problem in the clinic, with a poor prognosis and limited therapeutic options (25). Thus, urgent research action is required to aid in the identification of novel prognostic markers for HCC surveillance and to improve individual treatment approaches. A previous study has indicated that apoptosis-related proteins participate in the occurrence, development and metastasis of HCC (26). PED/PEA-15, a small cytosolic protein, is highly conserved in mammals and is ubiquitously expressed in human glucose metabolism. PED/PEA-15 participates in the modulation of numerous cellular processes, such as cell proliferation, death and survival (10). This enables PED/PEA-15 to interfere with intrinsic as well as extrinsic apoptosis pathways $(27,28)$. Previous studies have demonstrated that PED/PEA-15 is upregulated in a variety of tumors, such as breast and lung cancer, in which it facilitates tumor growth and is associated with poor survival outcomes $(9,29)$. By contrast, the upregulation of PED/PEA-15 in patients with ovarian cancer is associated with a good prognosis (30). This difference is primarily associated with the phosphorylation status of PED/PEA-15; in ovarian cancer, PED/PEA-15 is unphosphorylated (31). Therefore, PED/PEA-15 acts either as a tumor promoter or as a tumor suppressor, modulating cell proliferation or apoptosis depending on its phosphorylation status (11). Phosphorylated PED/PEA-15 is associated with increased ERK1/2 activity compared with 
Table IV. Associations between PED/PEA-15(S116) and P-p27(T187) levels in hepatocellular carcinoma determined by immunohistochemistry.

\begin{tabular}{|c|c|c|c|c|c|}
\hline \multirow[b]{2}{*}{ Marker } & \multirow[b]{2}{*}{$\mathrm{N}$} & \multicolumn{2}{|c|}{ P-p27(T187) expression } & \multirow[b]{2}{*}{ r-value } & \multirow[b]{2}{*}{ P-value } \\
\hline & & Positive & Negative & & \\
\hline \multicolumn{6}{|c|}{ PED/PEA-15(S116) expression } \\
\hline Positive & 41 & 36 & 5 & 0.434 & 0.001 \\
\hline Negative & 19 & 9 & 10 & & \\
\hline
\end{tabular}

PED/PEA-15(S116), phosphorylation of phosphoprotein enriched in diabetes/phosphoprotein enriched in astrocytes-15 at Ser116; P-p27(T187), phosphorylation of $\mathrm{p} 27$ at Thr187.

Table V. Univariate and multivariate analyses of prognostic variables for overall survival in patients with hepatocellular carcinoma.

\begin{tabular}{|c|c|c|c|c|c|c|}
\hline \multirow[b]{2}{*}{ Variable } & \multicolumn{3}{|c|}{ Univariate analysis } & \multicolumn{3}{|c|}{ Multivariate analysis } \\
\hline & HR & $95 \% \mathrm{CI}$ & P-value & HR & $95 \% \mathrm{CI}$ & P-value \\
\hline Age, years & 0.589 & $0.324-1.069$ & 0.082 & & & \\
\hline Sex & 1.193 & $0.648-2.198$ & 0.571 & & & \\
\hline $\mathrm{HBsAg}$ & 1.737 & $0.910-3.316$ & 0.094 & & & \\
\hline AFP & 0.584 & $0.319-1.069$ & 0.081 & & & \\
\hline Cirrhosis & 1.759 & $0.921-3.357$ & 0.087 & & & \\
\hline Tumor size, cm & 1.751 & $0.943-3.253$ & 0.076 & & & \\
\hline Tumor multiplicity & 0.269 & $0.132-0.549$ & $<0.001$ & & & \\
\hline TNM stage & 0.237 & $0.107-0.524$ & $<0.001$ & 2.782 & $1.136-6.802$ & 0.025 \\
\hline Edmondson grade & 0.226 & $0.116-0.440$ & $<0.001$ & & & \\
\hline Vascular invasion & 2.194 & $2.136-3.154$ & $<0.001$ & 3.255 & $0.996-10.635$ & 0.017 \\
\hline PED/PEA-15(S116) & 2.953 & $1.517-2.749$ & $<0.001$ & 5.801 & $2.472-13.609$ & 0.001 \\
\hline P-p27(T187) & 0.340 & $0.176-0.657$ & $<0.001$ & 2.063 & $0.924-4.605$ & 0.031 \\
\hline
\end{tabular}

HR, hazard ratio; HBsAg, hepatitis B virus surface antigen; AFP, $\alpha$-fetoprotein; TNM, tumor-node-metastasis; PED/PEA-15(S116), phosphorylation of phosphoprotein enriched in diabetes/phosphoprotein enriched in astrocytes-15 at Ser116; P-p27(T187), phosphorylation of p27 at Thr187.

that in non-tumorous cells, leading to increased cell growth and migration and, consequently, tumor promotion (32). According to the results of the present study, high expression levels of PED/PEA-15(S116) in HCC tissues were associated with a poor prognosis and a short survival time, which was consistent with the results of a previous study (33).

Several studies have reported that the suppression of p27 or its localization in the cytoplasm is associated with increased malignancy and a poor outcome in numerous types of cancer $(16,17,31)$. Uncontrolled cell proliferation is a characteristic of cancer and arises from the loss of control over the transition from the $G_{1}$ to $S$ phase in the cell cycle (17). P-p27(T187) triggers p27 degradation during the $G_{1} / S$ phase transition through the formation of the SCF ubiquitin ligase E3 complex, which results in the polyubiquitination of p27 (34). The suppression of p27 prevents efficient inhibition of cyclin-Cdk activities, and the resulting activity of these kinases facilitates cell proliferation (34). Alternatively, the suppression of p27 induces the deregulation of genes that serve a vital role in tumorigenesis and tumor metastasis (35). By contrast, when p27 is transported from the cell nucleus to the cytoplasm through phosphorylation, it alters the expression of genes modulated by p27 and exerts an oncogenic function by promoting cell movement and invasion through the modification of the cytoskeleton $(36,37)$. In the present study, P-p27(T187) protein was observed to be located in the cytoplasm and upregulated in HCC tissues compared with that in adjacent non-tumor and normal liver tissues. In addition, the positive expression of P-p27(T187) was associated with a shorter average survival time of patients with HCC, resulting in a poor prognosis. Therefore, we hypothesize that increased P-p27(T187) levels may affect HCC tumorigenesis and progression via nuclear export and polyubiquitination-mediated degradation of $\mathrm{p} 27$, which is consistent with previous findings.

The results of the present study also identified a positive association between PED/PEA-15(S116) and P-p27(T187) in HCC. Therefore, we hypothesized that PED/PEA-15(S116) 
A
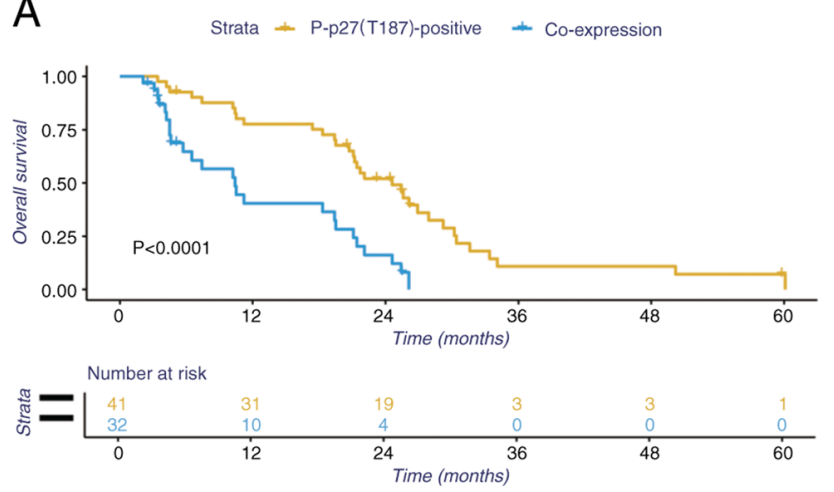

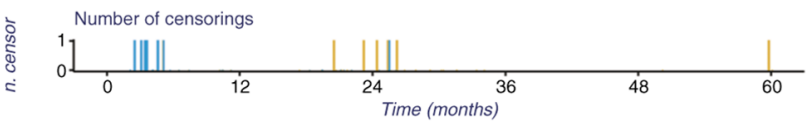
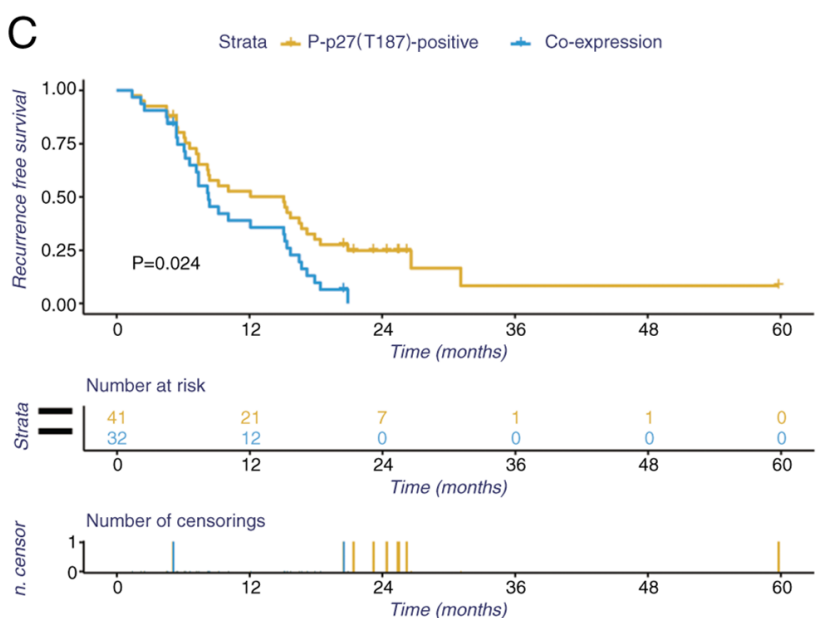

B
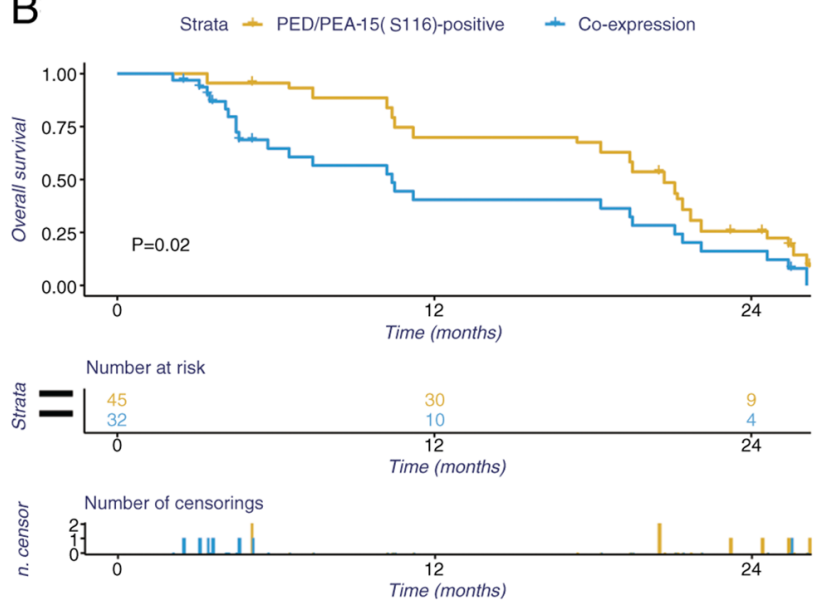

D Strata - PED/PEA-15( S116)-positive $\quad-$ Co-expression
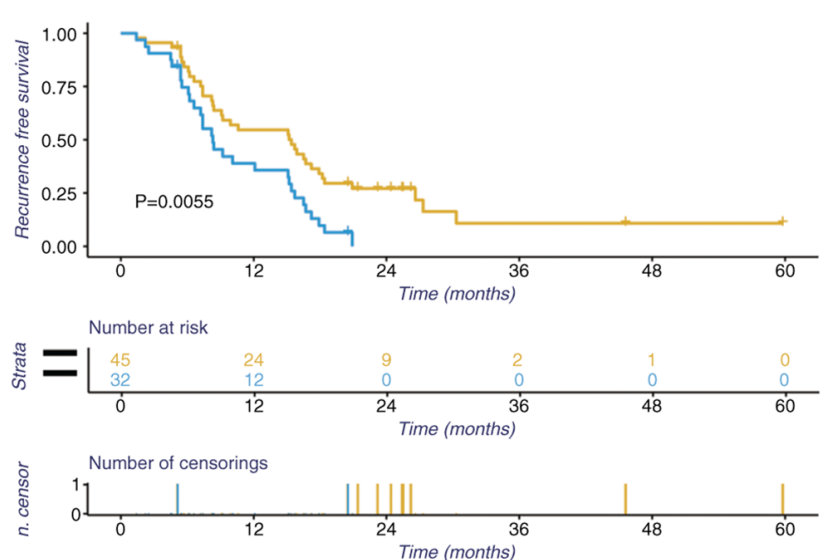

Figure 4. Prognostic value of co-expression of PED/PEA-15(S116) and P-p27(T187) in patients with HCC. (A and B) Kaplan-Meier analysis demonstrated differences in overall survival between patients with HCC positive for (A) P-p27(T187) and (B) PED/PEA-15(S116) with co-expression of P-p27(T187) and PED/PEA-15(S116). (C and D) A similar trend was observed for disease-free survival in patients with HCC positive for (C) P-p27(T187) and (D) PED/PEA-15(S116) with co-expression of P-p27(T187) and PED/PEA-15(S116). PED/PEA-15(S116), phosphorylation of phosphoprotein enriched in diabetes/phosphoprotein enriched in astrocytes-15 at Ser116; P-p27(T187), phosphorylation of p27 at Thr187; HCC, hepatocellular carcinoma.

may increase ERK phosphorylation, which regulates nucleocytoplasmic p27 export and polyubiquitination degradation, thus reducing the p27 protein level via P-p27(T187) $(21,38)$, and this phosphorylation cascade may serve an essential role in the MAPK/ERK pathway and contribute to the occurrence, development and prognosis of HCC.

The current study has several potential limitations. First, the number of samples was limited, which affected the power of the statistical analyses. Second, this was a retrospective study using medical data from a single institution; thus, certain inherent bias may exist. It is important that future prospective studies using larger sample sizes are conducted to validate the current findings. Finally, the present findings solely relied on histological examination and no further studies were performed for the two proteins; therefore, the phosphorylation cascade interactions in the MAPK/ERK pathway between $\mathrm{PED} / \mathrm{PEA}-15(\mathrm{~S} 116)$ and P-p27(T187) in in vivo and in vitro HCC models need to be further studied and validated.

In summary, the results of the present study suggested that PED/PEA-15(S116) and P-p27(T187) may be prospective prognostic biomarkers for HCC. However, further evidence is needed to validate their application. Based on the results of the current study, there was a significant association between the expression levels of PED/PEA-15(S116) and P-p27(T187) proteins in $\mathrm{HCC}$; however, their complicated interactions require additional research to determine their molecular mechanisms via the MAPK/ERK signaling pathway, which may unveil novel potential targets for HCC therapy.

\section{Acknowledgements}

Not applicable.

\section{Funding}

This work was funded by The Provincial Natural Science Foundation of Zhejiang (grant no. LY16H160004) and The Science Foundation of Zhejiang province (grant no. LQ18H160006).

\section{Availability of data and materials}

The datasets used and analyzed during the current study are available from the corresponding author on reasonable request. 


\section{Authors' contributions}

YW, SZ, XL and XX conceived the present study. YZ, XL, SZ and ZL drafted the manuscript. YW, MC, ZL, XZ, XL, SZ and $\mathrm{XX}$ made substantial contributions to the interpretation and analysis of the data, drafted the study and revised it critically for important intellectual content. YW and XX were major contributors in the revision of the manuscript. YW and XX confirm the authenticity of all the raw data. All authors read and approved the final manuscript.

\section{Ethics approval and consent to participate}

The study received ethical approval from the Research Ethics Committee of Yinzhou Hospital (Ningbo, China; approval no. 2017006). Written informed consent was obtained from all participants of the present study. The protocols of the present study regarding human subjects followed the ethical standards of the National Research Committee and the Declaration of Helsinki.

\section{Patient consent for publication}

Not applicable.

\section{Competing interests}

The authors declare that they have no competing interests.

\section{References}

1. Mittal S and El-Serag HB: Epidemiology of hepatocellular carcinoma: Consider the population. J Clin Gastroenterol 47 (Suppl): S2-S6, 2013

2. Bertuccio P, Turati F, Carioli G, Rodriguez T, La Vecchia C, Malvezzi M and Negri E: Global trends and predictions in hepatocellular carcinoma mortality. J Hepatol 67: 302-309, 2017.

3. Singal AG and El-Serag HB: Hepatocellular carcinoma from epidemiology to prevention: Translating knowledge into practice. Clin Gastroenterol Hepatol 13: 2140-2151, 2015.

4. Llovet JM, Zucman-Rossi J, Pikarsky E, Sangro B, Schwartz M, Sherman M and Gores: Hepatocellular carcinoma. Nat Rev Dis Primers 2: 16018, 2016.

5. Cai SH, Lu SX, Liu LL, Zhang CZ and Yun JP: Increased expression of hepatocyte nuclear factor 4 alpha transcribed by promoter 2 indicates a poor prognosis in hepatocellular carcinoma. Therap Adv Gastroenterol 10: 761-771, 2017.

6. Wong SS, Kim KM, Ting JC, Yu K, Fu J, Liu S, Cristescu R, Nebozhyn M, Gong L, Yue YG, et al: Genomic landscape and genetic heterogeneity in gastric adenocarcinoma revealed by whole-genome sequencing. Nat Commun 5: 5477, 2014.

7. Danziger N, Yokoyama M, Jay T, Cordier J, Glowinski J and Chneiweiss H: Cellular expression, developmental regulation, and phylogenic conservation of PEA-15, the astrocytic major phosphoprotein and protein kinase C substrate. J Neurochem 64: 1016-1025, 1995.

8. Greig FH and Nixon GF: Phosphoprotein enriched in astrocytes (PEA)-15: A potential therapeutic target in multiple disease states. Pharmacol Ther 143: 265-274, 2014.

9. Quintavalle C, Di Costanzo S, Zanca C, Tasset I, Fraldi A, Incoronato M, Mirabelli P, Monti M, Ballabio A, Pucci P, et al: Phosphorylation-regulated degradation of the tumor-suppressor form of PED by chaperone-mediated autophagy in lung cancer cells. J Cell Physiol 229: 1359-1368, 2014.

10. Xie X, Tang H, Pengliu, Kong Y, Wu M, Xiao X, Yang L, Gao J, Wei W, Lee J, et al: Development of PEA-15 using a potent non-viral vector for therapeutic application in breast cancer. Cancer Lett 356: 374-381, 2015.
11. Crespo-Flores SL, Cabezas A, Hassan S and Wei Y: PEA-15 C-terminal tail allosterically modulates death-effector domain conformation and facilitates protein-protein interactions. Int J Mol Sci 20: 3335, 2019.

12. Mace PD, Wallez Y, Egger MF, Dobaczewska MK, Robinson H, Pasquale EB and Riedl SJ: Structure of ERK2 bound to PEA-15 reveals a mechanism for rapid release of activated MAPK. Nat Commun 4: 1681, 2013.

13. Fiory F, Formisano P, Perruolo G and Beguinot F: Frontiers: PED/PEA-15, a multifunctional protein controlling cell survival and glucose metabolism. Am J Physiol Endocrinol Metab 297: E592-E601, 2009.

14. Besson A, Dowdy SF and Roberts JM: CDK inhibitors: Cell cycle regulators and beyond. Dev Cell 14: 159-169, 2008.

15. Bencivenga D, Caldarelli I, Stampone E, Mancini FP, Balestrieri ML, Della Ragione F and Borriello A: p27(Kip1) and human cancers: A reappraisal of a still enigmatic protein. Cancer Lett 403: 354-365, 2017.

16. Gupta A, Saltarski JM, White MA, Scaglioni PP and Gerber DE: Therapeutic targeting of nuclear export inhibition in lung cancer. J Thorac Oncol 12: 1446-1450, 2017.

17. Peng M, Wang J, Zhang D, Jin H, Li J, Wu XR and Huang C: PHLPP2 stabilization by p27 mediates its inhibition of bladder cancer invasion by promoting autophagic degradation of MMP2 protein. Oncogene 37: 5735-5748, 2018.

18. He W, Wang X, Chen L and Guan X: A crosstalk imbalance between p27(Kip1) and its interacting molecules enhances breast carcinogenesis. Cancer Biother Radiopharm 27: 399-402, 2012.

19. Grimmler M, Wang Y, Mund T, Cilensek Z, Keidel EM, Waddell MB, Jäkel H, Kullmann M, Kriwacki RW and Hengst L: Cdk-inhibitory activity and stability of p27Kip1 are directly regulated by oncogenic tyrosine kinases. Cell 128: 269-280, 2007.

20. Gallastegui E, Bicer A, Orlando S, Besson A, Pujol MJ and Bachs O: p27(Kip1) represses the Pitx2-mediated expression of p21(Cip1) and regulates DNA replication during cell cycle progression. Oncogene 36: 350-361, 2017.

21. Kim JE and Kang TC: Nucleocytoplasmic p27(Kip1) export is required for ERK1/2-mediated reactive astroglial proliferation following status epilepticus. Front Cell Neurosci 12: 152, 2018.

22. Zhou L, Rui JA, Ye DX, Wang SB, Chen SG and Qu Q: Edmondson-Steiner grading increases the predictive efficiency of TNM staging for long-term survival of patients with hepatocellular carcinoma after curative resection. World J Surg 32: 1748-1756, 2008.

23. Wang GK, Li SH, Zhao ZM, Liu SX, Zhang GX, Yang F, Wang Y, Wu F, Zhao XX and Xu ZY: Inhibition of heat shock protein 90 improves pulmonary arteriole remodeling in pulmonary arterial hypertension. Oncotarget 7: 54263-54273, 2016.

24. Andreozzi M, Quintavalle C, Benz D, Quagliata L, Matter M, Calabrese D, Tosti N, Ruiz C, Trapani F, Tornillo L, et al: HMGA1 expression in human hepatocellular carcinoma correlates with poor prognosis and promotes tumor growth and migration in in vitro models. Neoplasia 18: 724-731, 2016.

25. Chen SL, Liu LL, Lu SX, Luo RZ, Wang CH, Wang H, Cai SH, Yang X, Xie D, Zhang CZ and Yun JP: HBx-mediated decrease of AIM2 contributes to hepatocellular carcinoma metastasis. Mol Oncol 11: 1225-1240, 2017.

26. Morofuji N, Ojima H, Hiraoka N, Okusaka T, Esaki M, Nara S, Shimada K, Kishi Y and Kondo T: Antibody-based proteomics to identify an apoptosis signature for early recurrence of hepatocellular carcinoma. Clin Proteomics 13: 28, 2016.

27. Jiang X, Zhang C, Li W, Jiang D, Wei Z, Lv M, Xie X and Sun X: PEA-15 contributes to the clinicopathology and AKT-regulated cisplatin resistance in gastric cancer. Oncol Rep 41: 1949-1959, 2019.

28. Fiory F, Parrillo L, Raciti GA, Zatterale F, Nigro C, Mirra P, Falco R, Ulianich L, Di Jeso B, Formisano P, et al: PED/PEA-15 inhibits hydrogen peroxide-induced apoptosis in Ins-1E pancreatic beta-cells via PLD-1. PLoS One 9: e113655, 2014

29. Mohammed HN, Pickard MR and Mourtada-Maarabouni M: The protein phosphatase 4 - PEA15 axis regulates the survival of breast cancer cells. Cell Signal 28: 1389-1400, 2016.

30. Bartholomeusz C, Rosen D, Wei C, Kazansky A, Yamasaki F, Takahashi T, Itamochi H, Kondo S, Liu J and Ueno NT: PEA-15 induces autophagy in human ovarian cancer cells and is associated with prolonged overall survival. Cancer Res 68: 9302-9310, 2008. 
31. Lee J, Bartholomeusz C, Krishnamurthy S, Liu P, Saso H, Lafortune TA, Hortobagyi GN and Ueno NT: PEA-15 unphosphorylated at both serine 104 and serine 116 inhibits ovarian cancer cell tumorigenicity and progression through blocking $\beta$-catenin. Oncogenesis 1: e22, 2012.

32. Weijman JF, Riedl SJ and Mace PD: Structural studies of ERK2 protein complexes. Methods Mol Biol 1487: 53-63, 2017.

33. Quintavalle C, Hindupur SK, Quagliata L, Pallante P, Nigro C, Condorelli G, Andersen JB, Tagscherer KE, Roth W, Beguinot $\mathrm{F}$, et al: Phosphoprotein enriched in diabetes (PED/PEA15) promotes migration in hepatocellular carcinoma and confers resistance to sorafenib. Cell Death Dis 8: e3138, 2017.

34. Hoellein A, Graf S, Bassermann F, Schoeffmann S, Platz U, Holzlwimmer G, Kröger M, Peschel C, Oostendorp R, Quintanilla-Fend L and Keller U: Cks1 promotion of S phase entry and proliferation is independent of p27Kip1 suppression. Mol Cell Biol 32: 2416-2427, 2012.

35. Pippa R, Espinosa L, Gundem G, Garcia-Escudero R, Dominguez A, Orlando S, Gallastegui E, Saiz C, Besson A, Pujol MJ, et al: p27Kip1 represses transcription by direct interaction with $\mathrm{p} 130 / \mathrm{E} 2 \mathrm{~F} 4$ at the promoters of target genes. Oncogene 31: 4207-4220, 2012.
36. Besson A, Assoian RK and Roberts JM: Regulation of the cytoskeleton: An oncogenic function for CDK inhibitors? Nat Rev Cancer 4: 948-955, 2004

37. Besson A, Gurian-West M, Schmidt A, Hall A and Roberts JM p27Kip1 modulates cell migration through the regulation of RhoA activation. Genes Dev 18: 862-876, 2004.

38. Shin M, Lee KE, Yang EG, Jeon H and Song HK: PEA-15 facilitates EGFR dephosphorylation via ERK sequestration at increased ER-PM contacts in TNBC cells. FEBS Lett 589: 1033-1039, 2015.

This work is licensed under a Creative Commons Attribution-NonCommercial-NoDerivatives 4.0 International (CC BY-NC-ND 4.0) License. 